\title{
Development of a Clinical Decision Support System for Smart Algorithms in Emergency Medicine
}

\author{
Stefan VOGEL ${ }^{\mathrm{a}, 1}$, Andreas REISWICH ${ }^{\mathrm{b}}$, Zully RITTER ${ }^{\mathrm{a}}$, Michael SCHMUCKER ${ }^{\mathrm{b}}$, \\ Angela FUCHS ${ }^{d}$, Kerstin PISCHEK-KOCH ${ }^{\mathrm{a}}$, Stefanie WACHE ${ }^{\mathrm{a}}$, Katrin \\ ESSLINGER $^{\mathrm{d}}$, Michael DIETRICH ${ }^{\mathrm{c}}$, Tibor KESZTYÜS ${ }^{\mathrm{a}}$, Dagmar KREFTING ${ }^{\mathrm{a}}$, \\ Martin HAAG $^{\mathrm{b}}$ and Sabine BLASCHKE ${ }^{\mathrm{d}}$ \\ ${ }^{a}$ Department of Medical Informatics, University Medical Center Göttingen, Germany \\ ${ }^{\mathrm{b}}$ GECKO Institute, Heilbronn University of Applied Sciences, Germany \\ c German Research Center for Artificial Intelligence Berlin, Germany \\ ${ }^{d}$ Emergency Department, University Medical Center Göttingen, Germany
}

\begin{abstract}
The development of clinical decision support systems (CDSS) is complex and requires user-centered planning of assistive interventions. Especially in the setting of emergency care requiring time-critical decisions and interventions, it is important to adapt a CDSS to the needs of the user in terms of acceptance, usability and utility. In the so-called ENSURE project, a user-centered approach was applied to develop the CDSS intervention. In the context of this paper, we present a path to the first mockup development for a CDSS interface by addressing Campbell's Five Rights within the CDSS workflow.
\end{abstract}

Keywords. Clinical Decision Support System, Emergency Medicine, Artificial Intelligence, Clinical Process

\section{Introduction}

Preclinical and clinical emergency medicine has developed into a complex professional field in recent years $[1,2]$. One of the major problems represents the increase in the number of emergency patients presenting with a broad spectrum of illnesses and injuries ranging from outpatient care to intensive care [3]. Due to this problem, time-critical clinical decisions based on interdisciplinary medical knowledge represent the most important challenge in terms of patient safety and emergency care process efficiency. Clinical decision support systems (CDSS) are one approach to relieve clinicians, to contribute to patient safety and to improve clinical outcomes $[4,5]$. Similar to driver assistance in automobile clinical decision support systems can provide clinically relevant indications at the point of care to support the decision-making process of emergency physicians. The type of support can take many forms ranging from alerts in case of deterioration of vital signs to predictions of possible diagnoses and appropriate therapies.

\footnotetext{
${ }^{1}$ Corresponding Author, Stefan Vogel, University Medical Center Göttingen, Department of Medical Informatics, D-37075 Göttingen, Germany; E-mail: stefan.vogel@med.uni-goettingen.de.
} 
There are various technical options for implementing this support such as artificial intelligence (AI) approaches.

In the ENSURE project a prototypical CDSS to support timely and targeted diagnostics and initial therapy in emergency care will be developed and tested. It was the aim of the present work to design this CDSS intervention and develop initial software mock-ups for the future prototype.

\section{Methods}

The development of a CDSS is complex, costly and interdisciplinary [6]. For the development of the ENSURE prototype the user-centered design approach was applied [7]. To add value for the end users the Five Rights according to Campbell [8] were included in the planning of the CDSS intervention. Therefore, the following questions had to be clarified for the CDSS intervention: what is the right information, who is the right person, what is the right intervention format, what is the right channel and when is the right time in the workflow?

An essential part of the project is that two different information-processing methods will be implemented in the prototype. On the one hand a pattern-based approach based on the SOP book [10] and on the other hand a machine learning (ML) approach based on emergency data are implemented in order to support the diagnosis and initial therapy strategy. To evaluate the prototype, it is planned to randomly provide the user with one of the two approaches in a blind selection manner. In this way, it will be investigated whether one of the two approaches is superior in terms of performance and acceptance.

\section{Results}

In order to address the Five Rights and the user-centered design approach an experienced emergency physician took part in the conception phase. Based on the top 20 reasons for presentation in the Emergency Department (ED) [9] accounting for more than $80 \%$ of relevant emergencies, 30 emergency care algorithms resp. standard operating procedures (SOPs) as described by Blaschke and Walcher et al. [10] were identified.

Based on the SOPs 153 leading symptoms (in sum) associated to these 30 leading diagnoses were identified. The 153 leading symptoms were reduced to 96 in three iterations. Each iteration was based on the questions which leading symptoms are relevant to the 30 leading diagnoses, which information can be aggregated and whether there are symptom intersections between the leading diagnoses.

During emergency care this information is gathered and used for differential diagnosis. These aspects are of great importance for CDSS conception due to the fact that the intended diagnostic support has to process this gathered information. 


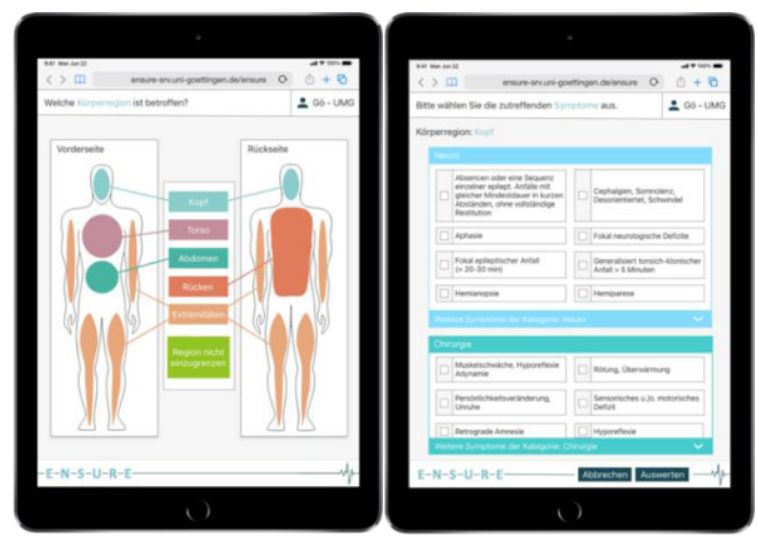

Figure 1. Mock-ups information aggregation of the CDSS prototype

Based on this preliminary work the first mock-ups (Fig. 1) were developed to allow users to collect information in a structured way using the future prototype. The 96 leading symptoms were assigned to five distinctive body regions as categories (head, thorax, abdomen, back, extremities). Another category "other" was introduced for symptoms that cannot be assigned to a specific body region. Furthermore, the symptoms were assigned to medical specialties to make the assessment intuitive and supportive in each emergency case situation.

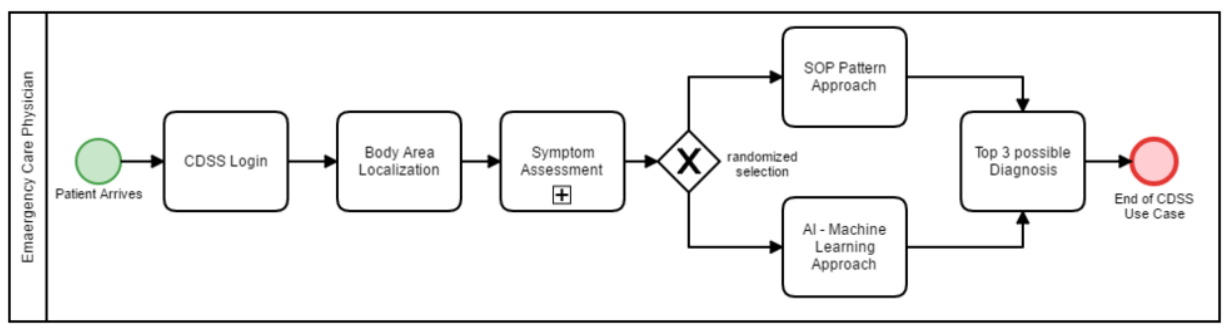

Figure 2. ENSURE CDSS prototype program flow created as BPMN with Camunda Modeler

Guided by the Five Rights and the user-centered design we were able to develop the prototypical program flow shown in Figure 2 and the first mock-ups in Figure 1.

\section{Discussion}

Emergency care is characterized by time-critical clinical decisions [11] to prevent possible long-term damage to patients or -in the worst case -even death. These conditions lead to a heavy burden on emergency physicians. Therefore, it is desirable to relieve this burden by providing a useful clinical supporting tool. Other research has shown that CDSS can have a positive impact in the emergency department [12]. To develop such a CDSS prototype, early involvement of experienced emergency physicians allows for a well-structured user-centered design.

In our research work, an SOP book with defined emergency care algorithms [10] was used for the development of a knowledge-based approach. The knowledge gained from this development will also be used in the development of an AI-based approach with ML algorithms. It is planned to develop different models for the ML approach and finally to use the model that has the best performance. Random forest models, support 
vector machines and artificial neural networks of different types are addressed in the development. The ML approach is developed in parallel on the basis of real emergency case data. Both approaches will be used in the prototype evaluation.

\section{Conclusion}

In this project, we develop a CDSS prototype for clinical emergency care. The conceptualized program flow of the prototype as well as the first mock-ups were created. The finalization of the ML approach and the linkage of the individual components to a complete prototype will follow.

The future evaluation of the prototype, especially with respect to the comparison of the knowledge-based and ML approaches, is a promising endeavor to gradually transition CDSS into routine emergency care.

\section{Acknowledgments}

The ENSURE project was funded by the Federal Ministry of Health Germany (BMG) (Grant No. 2520DAT803).

\section{References}

[1] Gemeinsamer Bundesausschuss 19.4.2018 „Regelungen zu einem gestuften System von Notfallstrukturen in Krankenhäusern gemäß §136c Absatz 4 SGB V“, https://www.gba.de/beschluesse/3301/, accessed 25.06.2020

[2] BMG, Reform der Notfallversorgung - schnellere Hilfe im Notfall“ 22.7.2019, https://www.bundesgesundheitsministerium.de/notfallversorgung.html?fbclid=IwAR3k0u_DH7W4 UEdzlgjamdFU-K3t4E0Ufyt3tcL9wPndVo6GMUYzMTETe9w, accessed 25.06.2020

[3] Schöpke T, Dodt C, Brachmann M, Schnieder W, Petersen PF, Böer J. Statusbericht aus deutschen Notaufnahmen. Notfall Rettungsmed 2014;17:660-670

[4] Sutton RT, Pincock D, Baumgart DC, et al. An overview of clinical decision support systems: benefits, risks, and strategies for success. npj Digit. Med. 2020;3:17

[5] Osheroff JA, Teich JM, Middleton B, Steen EB, Wright A, Detmer DE. A roadmap for national action on clinical decision support [published correction appears in J Am Med Inform Assoc. 2007 MayJun;14(3):389]. J Am Med Inform Assoc. 2007;14(2):141-145. doi:10.1197/jamia.M2334

[6] Richter J, Vogel S. Illustration of Clinical Decision Support System Development Complexity. Stud Health Technol Inform. 2020 Jun 26;272:261-264. doi: 10.3233/SHTI200544. PMID: 32604651.

[7] Kashfi H. Applying a user centered design methodology in a clinical context. Stud Health Technol Inform. 2010;160(2):927-31.

[8] Campbell RJ. The five rights of clinical decision support: CDS tools helpful for meeting meaningful use. Journal of AHIMA. 2013 Oct;84(10):42-7.

[9] Greiner F, Brammen D, et al.: Standardisierte Erhebung von Vorstellungsgründen in der Notaufnahme. Med Klin Intensivmed Notfmed 2018;113:115-123

[10] Blaschke S, Walcher F (Hrsg). SOP Handbuch Interdisziplinäre Notaufnahme. Medizinisch Wissenschaftliche Verlagsgesellschaft (MWV), Berlin, 1. Auflage 03/2015, 2. Auflage 2021 (in press)

[11] Al-Azri NH. How to think like an emergency care provider: a conceptual mental model for decision making in emergency care. Int J Emerg Med 2020;13:17.

[12] Bennett P, Hardiker NR. The use of computerized clinical decision support systems in emergency care: a substantive review of the literature. J Am Med Inform Assoc. 2017 May 1;24(3):655-668. doi: 10.1093/jamia/ocw151. PMID: 28031285; PMCID: PMC7651902. 